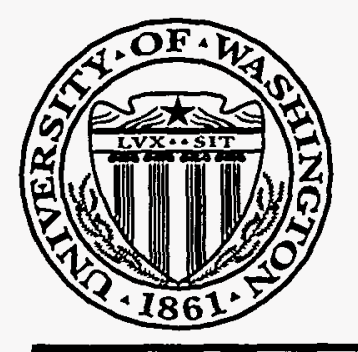

DOE/ ER/ $40537--54$
CONF-9509329-1

University of Washington Nuclear Physics Lab RECEIVED

$\operatorname{MAP} 181996$

OSTI

\title{
Preliminary Results from the Russian-American Gallium Experiment Cr-neutrino Source Measurement
}

S.R. Elliott ${ }^{1}$, J.N. Abdurashitov ${ }^{2}$, T.J. Bowles ${ }^{3}$, M.L. Cherry ${ }^{4}$, B.T. Cleveland ${ }^{5}$, T. Daily ${ }^{5}$, R Davis, Jr. ${ }^{5}$, V.N. Gavrin ${ }^{2}$, S. V. Girin ${ }^{2}$, V.V. Gorbatschev ${ }^{2}$, A.O. Gusev 2 , A.V. Kalikhov2, N.G. Khairnasov ${ }^{2}$, V.N.Karaulov ${ }^{6}$, T.V. Knodel ${ }^{2}$, V.N. Kornoukhov ${ }^{2}$, Yu.S.Khomyakov 7 , K. Lande ${ }^{5}$, C.K. Lee ${ }^{5}$, V.L.Levitin ${ }^{6}$, V.I.Maev ${ }^{6}$, I.N. Mirmov ${ }^{2}$, P.I.Nazarenko ${ }^{6}$, J.S. Nico ${ }^{3}$, A.M. Pshukov ${ }^{2}$, A.M Shalagin 2 , A.A. Shikhin ${ }^{2}$, V.S.Shkol'nik 6 , N.V.Skorikov 6 ,W.A. Teasdale $^{3}$, E.P. Veretenkin ${ }^{2}$, V.M. Vermul ${ }^{2}$, D.L. Wark ${ }^{3}{ }^{*}$, P.W. Wildenhain ${ }^{5}$, J.F. Wilkerson 1 , V. 'Yants ${ }^{2}$, G.T. Zatsepin ${ }^{2}$, and A.V.Zvonarev 7

${ }^{1}$ University of Washington, Seattle, WA 98195 USA

2Institute for Nuclear Research, Russian Academy of Sciences, Moscow 1173122, Russia $3^{3}$ Los Alamos National Laboratory, Los Alamos, NM 87545 USA

${ }^{4}$ Louisiana State University, Baton Rouge, LA 70803 USA

5 University of Pennsylvania, Philadelphia, PA 19104 USA

6Mangyshlak Atomic Energy Complex, Aktau, Kazakhstan Republik

${ }^{7}$ Power Physics Institute, Obninsk, Russia

Proceedings of the IV International Workshop on Theoretical and Phenomenological Aspects of Underground Physics, Toledo, Spain, September 17-21, 1995

Electroweak Interactions Group 


\section{Preliminary Results from the Russian-American Gallium Experiment Cr- neutrino Source Measurement}

S.R. Elliott ${ }^{1}$, J.N. Abdurashitov ${ }^{2}$, T.J. Bowles ${ }^{3}$, M.L. Cherry ${ }^{4}$, B.T. Cleveland ${ }^{5}$, T. Daily ${ }^{5}$, R Davis, Jr. 5 , V.N. Gavrin ${ }^{2}$, S. V. Girin ${ }^{2}$, V.V. Gorbatschev ${ }^{2}$, A.O. Gusev ${ }^{2}$, A.V. Kalikhov'2, N.G. Khairnasov ${ }^{2}$, V.N.Karaulov ${ }^{6}$, T.V. Knodel ${ }^{2}$, V.N. Kornoukhov ${ }^{2}$, Yu.S.Khomyakov ${ }^{7}, K$. Lande $^{5}$, C.K. Lee ${ }^{5}$, V.L.Levitin 6 , V.I.Maev ${ }^{6}$, I.N. Mirmov ${ }^{2}$, P.I.Nazarenko ${ }^{6}$, J.S. Nico ${ }^{3}$, A.M. Pshukov $^{2}$, A.M Shalagin ${ }^{2}$, A.A. Shikhin ${ }^{2}$, V.S.Shkol'nik ${ }^{6}$, N.V.Skorikov ${ }^{6}$, W.A. Teasdale ${ }^{3}$, E.P. Veretenkin $^{2}$, V.M. Vermul ${ }^{2}$, D.L. Wark ${ }^{3}{ }^{*}$, P.W. Wildenhain ${ }^{5}$, J.F. Wilkerson ${ }^{1}$, V. Yants ${ }^{2}$, G.T. Zatsepin ${ }^{2}$, and A.V.Zvonarev 7

${ }^{1}$ University of Washington, Seattle, WA 98195 USA

2Institute for Nuclear Research, Russian Academy of Sciences, Moscow 1173122, Russia

${ }^{3}$ Los Alamos National Laboratory, Los Alamos, NM 87545 USA

${ }^{4}$ Louisiana State University, Baton Rouge, LA 70803 USA

5University of Pennsylvania, Philadelphia, PA 19104 USA

6Mangyshlak Atomic Energy Complex, Aktau, Kazakhstan Republik

${ }^{7}$ Power Physics Institute, Obninsk, Russia

The Russian-American Gallium Experiment has been collecting solar neutrino data since early 1990. The flux measurement of solar neutrinos is well below that expected from solar models. We discuss the initial results of a measurement of experimental efficiencies by exposing the gallium target to neutrinos from an artificial source. The capture rate of neutrinos from this source is very close to that which is expected. The result can be expressed as a ratio of the measured capture rate to the anticipated rate from the source activity. This ratio is $0.93+0.15,-0.17$ where the systematic and statistical errors have been combined. To first order the experimental efficiencies are in agreement with those determined during solar neutrino measurements and in previous auxiliary measurements. One must conclude that the discrepancy between the measured solar neutrino flux and that predicted by the solar models can not arise from an experimental artifact.

\section{INTRODUCTION}

SAGE (the Russian-American Gallium Experiment) is a radiochemical solar neutrino experiment using ${ }^{71} \mathrm{Ga}$ as a target material. The low threshold $(233 \mathrm{keV})$ for inverse beta decay on this common isotope (40\% isotopic abundance of ${ }^{71} \mathrm{Ga}$ ) is well below the endpoint energy of the neutrino spectrum from proton-proton fusion. Thus gallium based experiments allow one to observe this low-energy branch of the solar neutrino spectrum. SAGE has been described in detail elsewhere (including these proceedings) and we direct the reader to the literature for details [1].
SAGE and the similar experiment GALLEX have been measuring the solar neutrino flux since the early 1990's. SAGE has observed a rate of $69 \pm 10+5,-7$ SNU [1] ( 1 SNU $=10^{-36}$ interactions/target atom/sec) and GALLEX has observed $77.1+9.6,-10.1$ SNU [2]. Both of these results are well below solar model predictions of $137+8,-7$ SNU [3] and $125 \pm 5$ SNU [4]. Taken together with other solar neutrino experimental results from the $\mathrm{Cl}$ experiment [5] and the Kamiokande experiment [6], a contradiction arises which cannot be accommodated by solar models [7-11].

The radiochemical experiments operate by chemically extracting and isolating a few atoms from many tons of target material (an isolation factor of about $10^{28}$ !). This impressively stringent requirement has raised skepticism 
about how well the extraction efficiency is known. The extraction efficiency has been determined by a variety of chemical and volumetric measurements which rely on the introduction and subsequent extraction of a known amount of stable Ge carrier to the gallium target. Although auxiliary measurements have confirmed this procedure [1], the direct calibration of the experiment with a well-characterized neutrino source would lend significant credibility to the radiochemical technique. This paper describes such a study of SAGE.

\section{THE SOURCE}

The decay of ${ }^{51} \mathrm{Cr}$ to ${ }^{51} \mathrm{~V}$ is via electron capture with a half life of 27.7 days and neutrino energies of $751 \mathrm{keV}(90 \%)$ and $426 \mathrm{keV}$ (10\%) [12]. A 320-keV gamma ray accompanies the $10 \%$ branch. These neutrino energies are well matched to that of proton-proton and ${ }^{7} \mathrm{Be}$ solar neutrinos. The source we have fabricated is $513 \mathrm{gm}$ of $92 \%$-enriched, ${ }^{50} \mathrm{Cr}$-metal rods encased in a tungsten shield which in turn is welded inside a stainless steel casing. The ${ }^{50} \mathrm{Cr}$ was irradiated and the source fabricated at the $\mathrm{BN}-350$ fast breeder reactor at Aktau, Kazakhstan.

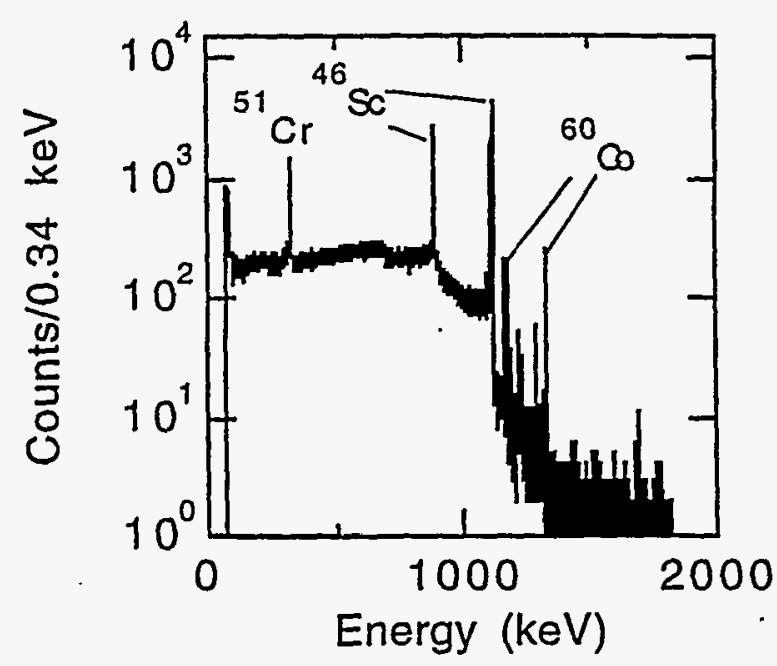

Figure 1. A Ge detector spectrum of the gamma rays emitted by the $\mathrm{Cr}$ source. Gamma lines are labeled by the isotope of origin. Other contaminants whose lines are not labeled include ${ }^{59} \mathrm{Fe},{ }^{182} \mathrm{Ta}$ and ${ }^{209} \mathrm{Sb}$.
The source was irradiated from September 4 to December 18, 1994. It was then transported to the Baksan Neutrino Observatory and exposure of the gallium began at 18:00 on December 26, 1994. At this time the source had an estimated activity of $509 \mathrm{kCi}$.

Fig. 1 shows a gamma spectrum of the source. The $320 \mathrm{keV}^{51} \mathrm{Cr}$ gamma is attenuated by a large factor by the tungsten shield but still shows a significant line in the spectrum. The higher energy lines of ${ }^{46} \mathrm{Sc},{ }^{59} \mathrm{Fe},{ }^{60} \mathrm{Co}$, and ${ }^{182}$ Ta have much smaller attenuations and thus produce lines even though they constitute far lower activity then the primary ${ }^{5 I_{C r}}$ source. Limits on the level of contamination activity can be determined from this spectrum. The $1.5 \mathrm{Ci}$ activity of ${ }^{46} \mathrm{Sc}$ is the largest single contribution and the total activity of all contaminants is estimated to be less than $2 \mathrm{Ci}$.

\section{EXTRACTION SCHEDULE}

The 55 tons of Ga employed at SAGE is distributed among 8 of 10 reactors with approximately 7 tons in each. All but one of these 10 reactors is equipped with the necessary mechanical equipment for the extraction process. The remaining reactor had the stirring mechanism removed permitting the storage of 13 tons of $\mathrm{Ga}$. This reactor was used for the $\mathrm{Cr}$ experiment. To perform the extraction with the usual chemical procedures, the gallium was transferred by a Teflon membrane pump to two of the other reactors after a $\mathrm{Cr}$ exposure. Eight source measurement extractions were conducted between Jan. 1 and May 24, 1995. The lengths of the exposure periods for the first 5 measurements were chosen so each would have about equal statistical sensitivity. The final 3 extractions were done monthly.

\section{SOURCE STRENGTH AND EXPECTED . COUNT RATE}

The decay of the ${ }^{51} \mathrm{Cr}$ deposits energy in the form of heat in the W/Cr cylinder. On average this energy is $35.51 \pm 0.16 \mathrm{keV} /$ decay [12]. Thus the generated heat provides a measure of the source activity by means of calorimetry. The $\mathrm{Cr}$ source is put inside a thermally isolated cavity inside the calorimeter. The temperature drop 
across a thermistor bridge joining this cavity is measured after thermal equilibrium has been reached. This temperature is compared with a calibration curve determined by a known heat source at many values spanning the range of the various ${ }^{51} \mathrm{Cr}$ measurements. The $\mathrm{Cr}$ activity was measured after each extraction with an average value determined for the source activity normalized to the date the source was first installed in the Ga.

The resulting activity is $509 \pm 10 \pm 40 \mathrm{kCi}$ with the first (second) uncertainty being due to statistics (systematics) The large systematic uncertainty is an overly conservative estimate of a possible error due to ignored non-linearities in the calorimeter. In the final analysis, this uncertainty should be greatly reduced by further direct calibration of the calorimeter.

The production rate of ${ }^{71} \mathrm{Ge}$ in the $\mathrm{Ga}$ can be factored into two contributions:

$$
\text { Rate }=\kappa<\mathrm{L}>\text { (atoms produced } / \mathrm{day} / \mathrm{kCi}) .
$$

The first ( $\kappa$ ) is due to the $\mathrm{Ga}$ density and neutrino interaction cross section. The second $(<L>)$ is the average neutrino path length through the gallium. Although the gallium nearly approximates a cylinder, the bottom of the $\mathrm{Ga}$ tank is dished and the value of $\langle\mathrm{L}\rangle$ is determined by Monte Carlo integration using an accurate map of the reactor shape. It is found to be $72.6 \pm 0.2 \mathrm{~cm}$.

The value of $\kappa$ is $0.398 \pm 0.040$ (neutrino captures/cm-day-MCi) where the dominate uncertainty arises due to the neutrino absorption cross section [13]. This uncertainty has been quoted at the $3 \sigma$ level. This cross section has recently been revisited by Hata and Haxton [14] who have reexamined the contribution due to excited states. They conclude that the previous estimate of this contribution inferred from $(p, n)$ reactions was unjustified and claim that the $\mathrm{Cr}$ source experiments themselves determine that contribution. We have not included this theoretical cross-section uncertainty in the neutrino capture cross section in our final uncertainty estimates.

Putting this all together gives an expected production rate on Dec. 26, 1994 at 18:00 of 14.7 \pm 1.5 atoms/day. This is equivalent to about
3500 SNU. The rate of production in the neighboring reactors is negligible and is ignored.

\section{WAVEFORM ANALYSIS}

The quantity of ${ }^{71} \mathrm{Ge}$ extracted from the gallium is determined by observing its decay in a small proportional counter (PC). When ${ }^{71_{\mathrm{Ge}}}$ undergoes electron capture decay, it emits $x$ rays of either $10.4 \mathrm{keV}$ (the $\mathrm{K}$ peak) or $1.2 \mathrm{keV}$ (the $L$ peak). The low energy of these $x$ rays results in a recoil electron of short range. Thus the radial extent of such electron trajectories in the counter is very short resulting in a PC pulse waveform with a fast risetime. Background processes, such as minimum ionizing particles, may deposit a similar amount of energy in the counter gas but will have large path lengths and hence slow risetimes.

A digitizer has been used since late 1992 to record waveforms from the proportional counters. Each waveform is digitized at a gigahertz and fit to a functional form which describes pulses in terms of the radial extent of the trajectories in a PC [15]. The desired fast risetime events which would result from the ${ }^{71} \mathrm{Ge} x$ rays and Auger electrons will have a very small radial extent. Although this technique and the hardware risetime measurement technique [1] differ little for the large-amplitude $\mathrm{K}$-peak waveforms, it is much more powerful in the lowamplitude L-peak region. In this region, offsets, noise, and nonlinearities of electronic modules dramatically decrease the hardware-based background rejection. The waveform analysis, however, overcomes these difficulties to provide a good separation between signal and background. The results here represent the first presentation of L-peak data from the SAGE experiment.

\section{ANALYSIS}

Each extraction has its candidate ${ }^{71_{G e}}$ events chosen by selection in the energyrisetime plane. The time structure of these events is then analyzed with a maximum likelihood method [16] to separate the ${ }^{71_{G e}}$ 11.4-day decay from a constant rate background. The only difference between this analysis and that done for the solar neutrino runs is that one 
must account for the decay of the ${ }^{51} \mathrm{Cr}$ (as opposed to a constant solar flux), a "background" contribution from solar neutrinos, and a carryover correction arising from the ${ }^{71} \mathrm{Ge}$ that is not removed because of the approximately $15 \%$ inefficiency of the preceding chemical extraction.

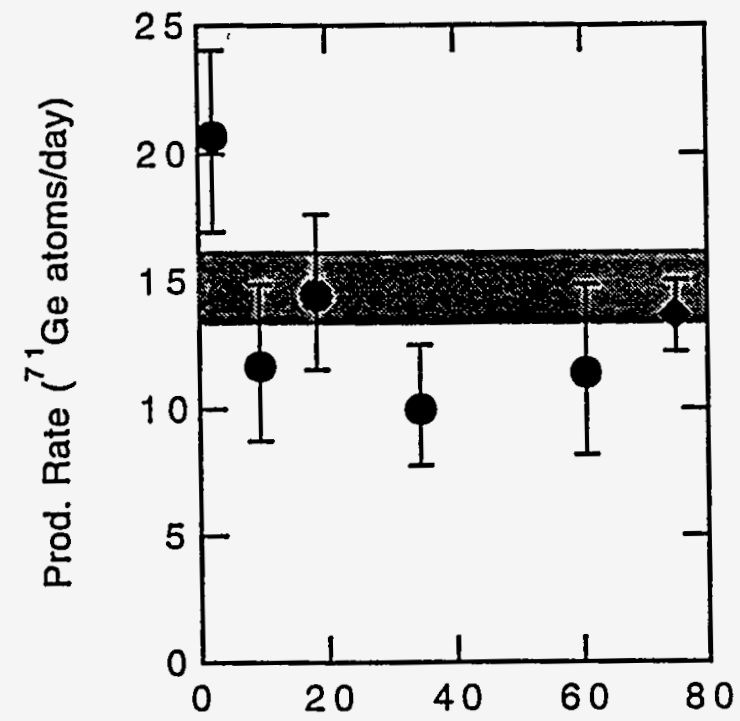

Extr. (day of 1995 year)

Figure 2. The 5 production rate measurements. The solid circles indicate the 5 measurements and the diamond the combined result. The shaded bar indicates the range of the expected production rate defined by its uncertainty.

Figure 2 shows the results of the first 5 exposure/extractions which are being presented here. The result of each run is normalized to the Cr activity production rate on Dec. 26 at 16:00. The combined fit of the 5 runs gives a production rate of $13.6 \pm 1.4+0.9,-1.4$ atoms/day where the first uncertainty is statistical and the latter is systematic. A fit permitting the ${ }^{71} \mathrm{Ge}$ half life to vary gives $12.0 \pm 1.5$ days also consistent with its known half life of 11.4 days. The analysis of the final 3 runs will modestly decrease the statistical uncertainty. Figure 3 shows the energy spectrum of the fast events clearly indicating the $\mathrm{K}$ and $\mathrm{L}$ peaks.

Table 1 lists the preliminary estimates of the uncertainties associated with these measurements. For the most part they are determined in a similar manner as the solar neutrino runs [1].
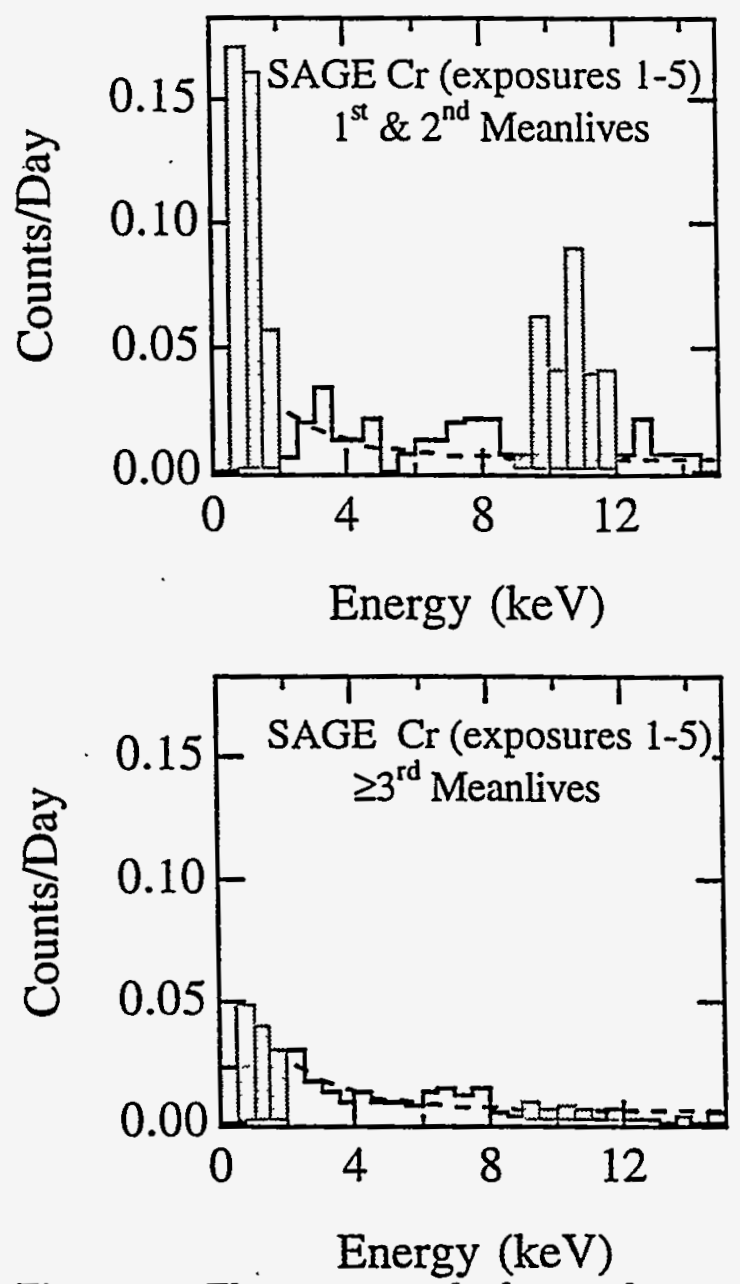

Figure 3. The top panel shows the energy spectrum of the fast risetime events observed during the $5 \mathrm{Cr}$ exposure measurements during the first two meanlives of counting. The bottom panel show a similar spectrum but for counting after two meanlives. The ${ }^{71} \mathrm{Ge} \mathrm{K}$ and $\mathrm{L}$ peaks are evident in the top panel.

Table 1. A summary of the contributions to the systematic uncertainty. The total is taken to be the quadrature sum of the contributions. The statistical uncertainty is $\pm 10 \%$.

\begin{tabular}{lc}
\hline \hline Chem. Extr. Efficiency & $\pm 5.5 \%$ \\
Counting Efficiency & $+2.9,-4.2 \%$ \\
Radon & $-7.7 \%$ \\
Solar Neutrino Subtraction & $\pm 0.3 \%$ \\
Carryover Ge Subtraction & $\pm 1.6 \%$ \\
\hline Total Systematic & $+6.4,-10.5 \%$ \\
\hline \hline
\end{tabular}




\section{DISCUSSION}

The final result can be expressed as a ratio of the measured ${ }^{71} \mathrm{Ge}$ production to that expected due to the source strength. The result of 0.93 $+0.15,-0.17$ is consistent with 1.0 to first order. Although a finer level of precision will be determined as the final analysis is completed, the result indicates that the experimental efficiencies are as previously claimed [1]. One concludes therefore that the discrepancy between the SAGE measured solar neutrino flux and the solar model predication cannot be explained by an experimental artifact.

The experimental efficiencies are known to a higher precision than can be tested by this method. Thus in an absolute sense, this measurement does not represent a direct calibration. A more precise description is that the $\mathrm{Cr}$ experiment is a systematic test of the experimental procedures. The solar flux measurement will not be scaled by the above ratio.

GALLEX has completed a ${ }^{51} \mathrm{Cr}$ calibration measurement with the result $0.97 \pm 0.11$ [17]. Both of these experiments give similar solst neutrino flux results and have verified their efficiencies even though they employ very different chemistries, indicating that it is very difficult to dismiss the experimental results and their striking disagreement with standard solar model predictions.

\section{ACKNOWLEDGMENTS}

The SAGE collaboration wishes to thank A. E. Chudakov, M. A. Markov, V. A. Matveev, V. A. Rubakov, and A. N. Tavkhelidze for their continued interest in out work and for stimulating discussions. We are also grateful to R. G. H. Robertson, A. Yu. Smirnov, and many members of the GALLEX collaboration for useful discussions. We acknowledge the support of the Russian Academy of Sciences, the Institute for Nuclear Research of the Russian Academy of Sciences, the Russian Ministry of Science and Technology, the Russian Foundation of Fundamental Research, the Division of Nuclear Physics of the Department of Energy, and the National Science
Foundation. The research described in this publication was made possible in part by Grant .M7F000 from the International Science Foundation and Grant M7F300 from the International Science Foundation and Russian Goverment.

\section{REFERENCES}

* present address: Department of Particle and Nuclear Physics, Oxford University, Keble Road, Oxford OX1 3RH, UK.

1. J. N. Abdurashitov et al., Phys. Lett. B357, 237 (1995); and these proceedings.

2. P. Anselmann et al., Phys. Lett. B327, 377 (1994).

3. J. N. Bahcall, and M. Pinsonneault, Rev. Mod. Phys., Oct. 1995, in press.

4. S. Turck-Chieze and I. Lopes, Astrophys. J. 408, 347 (1993).

5. B. T. Cleveland et al., Nucl. Phys. (proceed. supl.) B38, 47 (1995).

6. Y. Suzuki, Nucl. Phys. (proceed. supl.) B38, 54 (1995).

J. IJ. Bahcall, Phys. Lett. B338, 276 (1994).

8. V. Berezinsky, Comm. Nucl. Part. Phys. 21, 249 (1994).

9. S. Parke, Phys. Rev. Lett. 74, 839 (1995).

10. N. Hata, S. Bludman, and P. Langacker, Phys. Rev. D49, 3622 (1994).

11. V. Castellani et al., Phys. Rev. D50, 4749 (1994).

12. U. Schotzig and H. Schrader, Halbwertszeiten und PhotonenEmissions-wahrscheinlichkeiten von haufig verwendeten Radionukliden, PTB-Bericht PTB-Ra-16/4, Braunschweig; 1993.

13. J. N. Bahcall and R. K. Ulrich, Rev. Mod. Phys. 60, 297 (1988).

14. Naoya Hata and Wich Haxton, Phys. Lett. B353, 422 (1995).

15. S. R. Elliott, Nucl. Instrum. and Meth. A290, 158 (1990)

16. B. T. Cleveland, Nucl. Instrum. Methods 214, 451 (1983).

17. P. Anselmann et al., Phys. Lett. B342, 440 (1995); and these proceedings. 


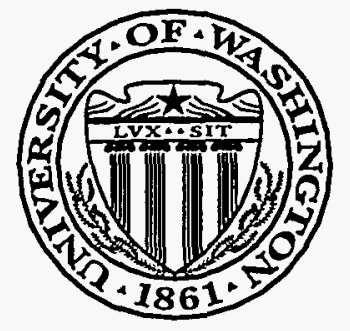

University of Washington Nuclear Physics Lab

\section{Preliminary Results from the Russian-American Gallium Experiment Cr-neutrino Source Measurement}

S.R. Elliott ${ }^{1}$, J.N. Abdurashitov ${ }^{2}$, T.J. Bowles ${ }^{3}$, M.L. Cherry ${ }^{4}$, B.T. Cleveland ${ }^{5}$, T. Daily ${ }^{5}$, R Davis, Jr. ${ }^{5}$, V.N. Gavrin ${ }^{2}$, S. V. Girin ${ }^{2}$, V.V. Gorbatschev ${ }^{2}$, A.O. Gusev ${ }^{2}$, A.V. Kalikhov ${ }^{2}$, N.G. Khairnasov ${ }^{2}$, V.N.Karaulov 6 , T.V. Knodel ${ }^{2}$, V.N. Kornoukhov ${ }^{2}$, Yu.S.Khomyakov ${ }^{7}$, K. Lande ${ }^{5}$, C.K. Lee ${ }^{5}$, V.L.Levitin ${ }^{6}$, V.I.Maev ${ }^{6}$, I.N. Mirmov ${ }^{2}$, P.I.Nazarenko6, J.S. Nico ${ }^{3}$, A.M. Pshukov' ${ }^{2}$, A.M Shalagin ${ }^{2}$, A.A. Shikhin ${ }^{2}$, V.S.Shkol'nik ${ }^{6}$, N.V.Skorikov6,W.A. Teasdale $^{3}$, E.P. Veretenkin ${ }^{2}$, V.M. Vermul ${ }^{2}$, D.L. Wark ${ }^{3}{ }^{*}$, P.W. Wildenhain ${ }^{5}$, J.F. Wilkerson $^{1}$, V. Yants ${ }^{2}$, G.T. Zatsepin ${ }^{2}$, and A.V.Zvonarev 7

${ }^{1}$ University of Washington, Seattle, WA 98195 USA

${ }^{2}$ Institute for Nuclear Research, Russian Academy of Sciences, Moscow 1173122, Russia

3 Los Alamos National Laboratory, Los Alamos, NM 87545 USA

${ }^{4}$ Louisiana State University, Baton Rouge, LA 70803 USA

5University of Pennsylvania, Philadelphia, PA 19104 USA ${ }^{6}$ Mangyshlak Atomic Energy Complex, Aktau, Kazakhstan Republik

7Power Physics Institute, Obninsk, Russia

Proceedings of the $I V$ International Workshop on Theoretical and Phenomenological Aspects of Underground Physics, Toledo, Spain, September 17-21, 1995

Electroweak Interactions Group 
Preliminary Results from the Russian-American Gallium Experiment Crneutrino Source Measurement

S.R. Elliott ${ }^{1}$, J.N. Abdurashitov ${ }^{2}$, T.J. Bowles ${ }^{3}$, M.L. Cherry ${ }^{4}$, B.T. Cleveland ${ }^{5}$, T. Daily $^{5}$, R Davis, Jr. ${ }^{5}$, V.N. Gavrin ${ }^{2}$, S. V. Girin ${ }^{2}$, V.V. Gorbatschev ${ }^{2}$, A.O. Gusev ${ }^{2}$, A.V. Kalikhov ${ }^{2}$, N.G. Khairnasov ${ }^{2}$, V.N.Karaulov 6 , T.V. Knodel ${ }^{2}$, V.N. Kornoukhov ${ }^{2}$, Yu.S.Khomyakov ${ }^{7}, \mathrm{~K}$. Lande $^{5}$, C.K. Lee ${ }^{5}$, V.L.Levitin ${ }^{6}$, V.I.Maev ${ }^{6}$, I.N. Mirmov ${ }^{2}$, P.I.Nazarenko ${ }^{6}$, J.S. Nico ${ }^{3}$, A.M. Pshukov $^{2}$, A.M Shalagin ${ }^{2}$, A.A. Shikhin ${ }^{2}$, V.S.Shkol'nik ${ }^{6}$, N.V.Skorikov ${ }^{6}$,W.A. Teasdale ${ }^{3}$, E.P. Veretenkin $^{2}$, V.M. Vermul ${ }^{2}$, D.L. Wark ${ }^{3}{ }^{*}$, P.W. Wildenhain ${ }^{5}$, J.F. Wilkerson ${ }^{1}$, V. Yants ${ }^{2}$, G.T. Zatsepin ${ }^{2}$, and A.V.Zvonarev 7

\author{
${ }^{1}$ University of Washington, Seattle, WA 98195 USA \\ 2 Institute for Nuclear Research, Russian Academy of Sciences, Moscow 1173122, Russia \\ ${ }^{3}$ Los Alamos National Laboratory, Los Alamos, NM 87545 USA \\ ${ }^{4}$ Louisiana State University, Baton Rouge, LA 70803 USA \\ 5 University of Pennsylvania, Philadelphia, PA 19104 USA \\ ${ }^{6}$ Mangyshlak Atomic Energy Complex, Aktau, Kazakhstan Republik \\ 7 Power Physics Institute, Obninsk, Russia
}

The Russian-American Gallium Experiment has been collecting solar neutrino data since early 1990. The flux measurement of solar neutrinos is well below that expected from solar models. We discuss the initial results of a measurement of experimental efficiencies by exposing the gallium target to neutrinos from an artificial source. The capture rate of neutrinos from this source is very close to that which is expected. The result can be expressed as a ratio of the measured capture rate to the anticipated rate from the source activity. This ratio is $0.93+0.15,-0.17$ where the systematic and statistical errors have been combined. To first order the experimental efficiencies are in agreement with those determined during solar neutrino measurements and in previous auxiliary measurements. One must conclude that the discrepancy between the measured solar neutrino flux and that predicted by the solar models can not arise from an experimental artifact.

\section{INTRODUCTION}

SAGE (the Russian-American Gallium Experiment) is a radiochemical solar neutrino

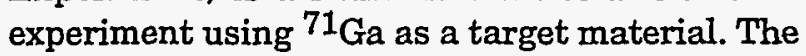
low threshold ( $233 \mathrm{keV}$ ) for inverse beta decay on this common isotope ( $40 \%$ isotopic abundance of ${ }^{71} \mathrm{Ga}$ ) is well below the endpoint energy of the neutrino spectrum from proton-proton fusion. Thus gallium based experiments allow one to observe this low-energy branch of the solar neutrino spectrum. SAGE has been described in detail elsewhere (including these proceedings) and we direct the reader to the literature for details [1].
SAGE and the similar experiment GALLEX have been measuring the solar neutrino flux since the early 1990's. SAGE has observed a rate of $69 \pm 10+5,-7$ SNU [1] (1 SNU $=10^{-36}$ interactions/target atom/sec) and GALLEX has observed 77.1 +9.6,-10.1 SNU [2]. Both of these results are well below solar model predictions of $137+8,-7$ SNU [3] and $125 \pm 5$ SNU [4]. Taken together with other solar neutrino experimental results from the $\mathrm{Cl}$ experiment [5] and the Kamiokande experiment [6], a contradiction arises which cannot be accommodated by solar models [7-11].

The radiochemical experiments operate by chemically extracting and isolating a few atoms from many tons of target material (an isolation factor of about $10^{28}$ !). This impressively stringent requirement has raised skepticism 
about how well the extraction efficiency is known. The extraction efficiency has been determined by a variety of chemical and volumetric measurements which rely on the introduction and subsequent extraction of a known amount of stable Ge carrier to the gallium target. Although auxiliary measurements have confirmed this procedure [1], the direct calibration of the experiment with a well-characterized neutrino source would lend significant credibility to the radiochemical technique. This paper describes such a study of SAGE.

\section{THE SOURCE}

The decay of ${ }^{51} \mathrm{Cr}$ to ${ }^{51} \mathrm{~V}$ is via electron capture with a half life of 27.7 days and neutrino energies of $751 \mathrm{keV}(90 \%)$ and $426 \mathrm{keV}$ (10\%) [12] A $320-\mathrm{keV}$ gamma ray accompanies the $10 \%$ branch. These neutrino energies are well matched to that of proton-proton and ${ }^{7} \mathrm{Be}$ solar neutrinos. The source we have fabricated is $513 \mathrm{gm}$ of $92 \%$-enriched, ${ }^{50} \mathrm{Cr}$-metal rods encased in a tungsten shield which in turn is welded inside a stainless steel casing. The ${ }^{50} \mathrm{Cr}$ was irradiated and the source fabricated at the BN-350 fast breeder reactor at Aktau, Kazakhstan.

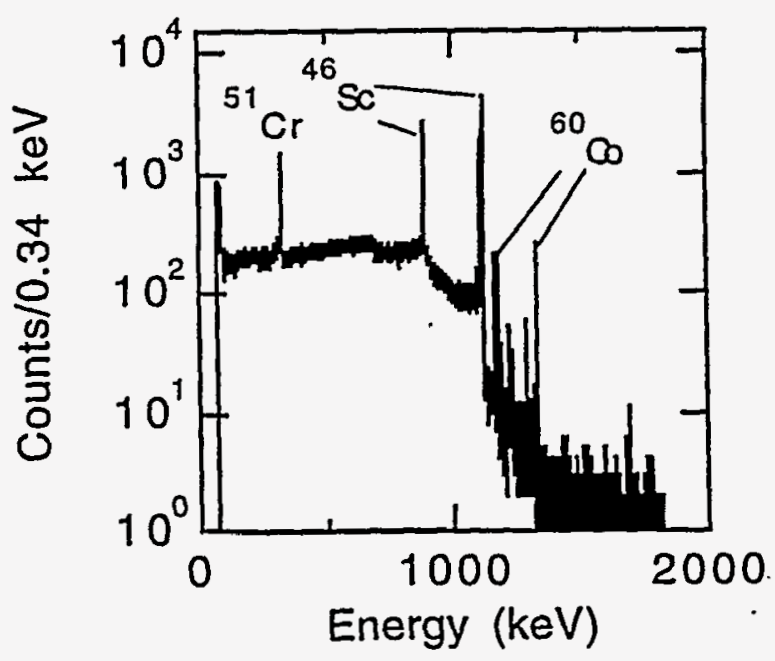

Figure 1. A Ge detector spectrum of the gamma rays emitted by the $\mathrm{Cr}$ source. Gamma lines are labeled by the isotope of origin. Other contaminants whose lines are not labeled include $59 \mathrm{Fe},{ }^{182} \mathrm{Ta}$ and ${ }^{209} \mathrm{Sb}$.
The source was irradiated from September 4 to December 18, 1994. It was then transported to the Baksan Neutrino Observatory and exposure of the gallium began at 18:00 on December 26, 1994. At this time the source had an estimated activity of $509 \mathrm{kCi}$.

Fig. 1 shows a gamma spectrum of the source. The $320 \mathrm{keV}^{51} \mathrm{Cr}$ gamma is attenuated by a large factor by the tungsten shield but still shows a significant line in the spectrum. The higher energy lines of ${ }^{46} \mathrm{Sc},{ }^{59} \mathrm{Fe},{ }^{60} \mathrm{Co}$, and ${ }_{182} \mathrm{Ta}$ have much smaller attenuations and thus produce lines even though they constitute far lower activity then the primary ${ }^{51} \mathrm{Cr}$ source. Limits on the level of contamination activity can be determined from this spectrum. The $1.5 \mathrm{Ci}$ activity of ${ }^{46} \mathrm{Sc}$ is the largest single contribution and the total activity of all contaminants is estimated to be less than $2 \mathrm{Ci}$.

\section{EXTRACTION SCHEDULE}

The 55 tons of Ga employed at SAGE is distributed among 8 of 10 . reactors with approximately 7 tons in each. All but one of these 10 reactors is equipped with the necessary mechanical equipment for the extraction process. The remaining reactor had the stirring mechanism removed permitting the storage of 13 tons of $\mathrm{Ga}$. This reactor was used for the $\mathrm{Cr}$ experiment. To perform the extraction with the usual chemical procedures, the gallium was transferred by a Teflon membrane pump to two of the other reactors after a $\mathrm{Cr}$ exposure. Eight source measurement extractions were conducted between Jan. I and May 24, 1995. The lengths of the exposure periods for the first 5 measurements were chosen so each would have about equal statistical sensitivity. The final 3 extractions were done monthly.

\section{SOURCE STRENGTH AND EXPECTED COUNT RATE}

The decay of the ${ }^{51} \mathrm{Cr}$ deposits energy in the form of heat in the W/Cr cylinder. On average this energy is $35.51 \pm 0.16 \mathrm{keV} /$ decay [12]. Thus the generated heat provides a measure of the source activity by means of calorimetry. The $\mathrm{Cr}$ source is put inside a thermally isolated cavity inside the calorimeter. The temperature drop 
across a thermistor bridge joining this cavity is measured after thermal equilibrium has been reached. This temperature is compared with a calibration curve determined by a known heat source at many values spanning the range of the various ${ }^{51} \mathrm{Cr}$ measurements. The $\mathrm{Cr}$ activity was measured after each extraction with an average value determined for the source activity normalized to the date the source was first installed in the Ga.

The resulting activity is $509 \pm 10 \pm 40 \mathrm{kCi}$ with the first (second) uncertainty being due to statistics (systematics) The large systematic uncertainty is an overly conservative estimate of a possible error due to ignored non-linearities in the calorimeter. In the final analysis, this uncertainty should be greatly reduced by further direct calibration of the calorimeter.

The production rate of ${ }^{71} \mathrm{Ge}$ in the $\mathrm{Ga}$ can be factored into two contributions:

$$
\text { Rate }=\kappa<\mathrm{L}>\text { (atoms produced } / \mathrm{day} / \mathrm{kCi}) .
$$

The first ( $\kappa$ ) is due to the Ga density and neutrino interaction cross section. The second $(\langle L\rangle)$ is the average neutrino path length through the gallium. Although the gallium nearly approximates a cylinder, the bottom of the $\mathrm{Ga}$ tank is dished and the value of $\langle\mathrm{L}\rangle$ is determined by Monte Carlo integration using an accurate map of the reactor shape. It is found to be $72.6 \pm 0.2 \mathrm{~cm}$.

The value of $\kappa$ is $0.398 \pm 0.040$ (neutrino captures/cm-day-MCi) where the dominate uncertainty arises due to the neutrino absorption cross section [13]. This uncertainty has been quoted at the $3 \sigma$ level. This cross section has recently been revisited by Hata and Haxton [14] who have reexamined the contribution due to excited states. They conclude that the previous estimate of this contribution inferred from $(p, n)$ reactions was unjustified and claim that the $\mathrm{Cr}$ source experiments - themselves determine that contribution. We have not included this theoretical cross-section uncertainty in the neutrino capture cross section in our final uncertainty estimates.

Putting this all together gives an expected production rate on Dec. 26, 1994 at 18:00 of 14.7 \pm 1.5 atoms/day. This is equivalent to about
3500 SNU. The rate of production in the neighboring reactors is negligible and is ignored.

\section{WAVEFORM ANALYSIS}

The quantity of ${ }^{71_{\mathrm{Ge}}}$ extracted from the gallium is determined by observing its decay in a small proportional counter (PC). When ${ }^{71} \mathrm{Ge}$ undergoes electron capture decay, it emits $\mathrm{x}$ rays of either $10.4 \mathrm{keV}$ (the $\mathrm{K}$ peak) or $1.2 \mathrm{keV}$ (the $L$ peak). The low energy of these $x$ rays results in a recoil electron of short range. Thus the radial extent of such electron trajectories in the counter is very short resulting in a PC pulse waveform with a fast risetime. Background processes, such as minimum ionizing particles, may deposit a similar amount of energy in the counter gas but will have large path lengths and hence slow risetimes.

A digitizer has been used since late 1992 to record waveforms from the proportional counters. Each waveform is digitized at a gigahertz and fit to a functional form which describes pulses in terms of the radial extent of the trajectories in a PC [15]. The desired fast risetime events which would result from the ${ }^{71} \mathrm{Ge} x$ rays and Auger electrons will have a very small radial extent. Although this technique and the hardware risetime measurement technique [1] differ little for the large-amplitude K-peak waveforms, it is much more powerful in the lowamplitude L-peak region. In this region, offsets, noise, and nonlinearities of electronic modules dramatically decrease the hardware-based background rejection. The waveform analysis, however, overcomes these difficulties to provide a good separation between signal and background. The results here represent the first presentation of L-peak data from the SAGE experiment.

\section{ANALYSIS}

Each extraction has its candidate ${ }^{71_{\mathrm{Ge}}}$ events chosen by selection in the energyrisetime plane. The time structure of these events is then analyzed with a maximum likelihood method [16] to separate the ${ }^{71_{G e}}$ 11.4-day decay from a constant rate background. The only difference between this analysis and that done for the solar neutrino runs is that one 
must account for the decay of the ${ }^{51} \mathrm{Cr}$ (as opposed to a constant solar flux), a "background" contribution from solar neutrinos, and a carryover correction arising from the ${ }^{71} \mathrm{Ge}$ that is not removed because of the approximately $15 \%$ inefficiency of the preceding chemical extraction.

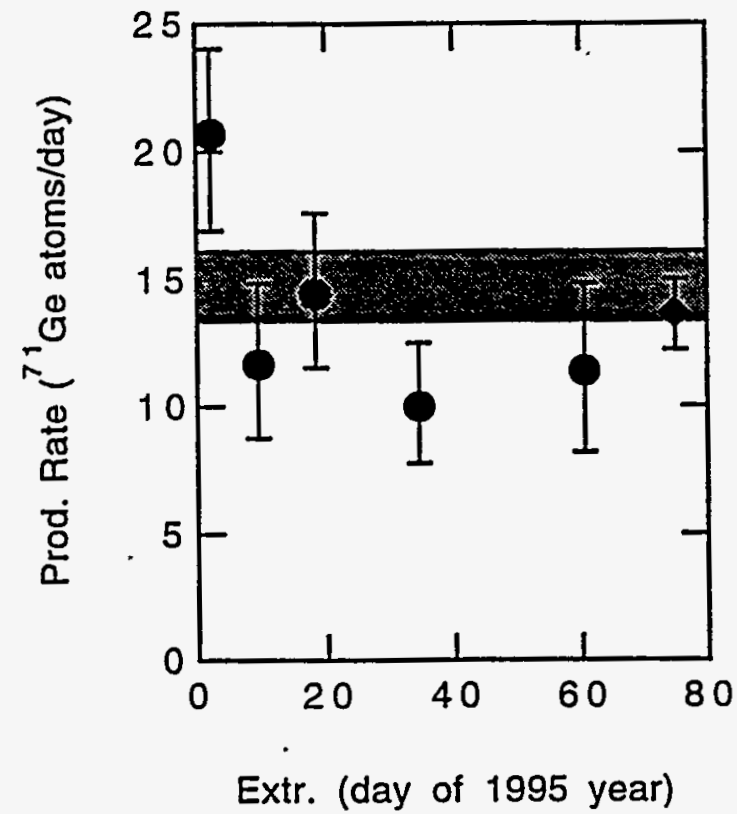

Figure 2. The 5 production rate measurements. The solid circles indicate the 5 measurements and the diamond the combined result. The shaded bar indicates the range of the expected production rate defined by its uncertainty.

Figure 2 shows the results of the first 5 exposure/extractions which are being presented here. The result of each run is normalized to the Cr activity production rate on Dec. 26 at 16:00. The combined fit of the 5 runs gives a production rate of $13.6 \pm 1.4+0.9,-1.4$ atoms/day where the first uncertainty is statistical and the latter is systematic. A fit permitting the ${ }^{71_{G e}}$ half life to vary gives $12.0 \pm 1.5$ days also consistent with its known half life of 11.4 days. The analysis of the final 3 runs will modestly decrease the statistical uncertainty. Figure 3 shows the energy spectrum of the fast events clearly indicating the $\mathrm{K}$ and $\mathrm{L}$ peaks.

Table 1 lists the preliminary estimates of the uncertainties associated with these measurements. For the most part they are determined in a similar manner as the solar neutrino runs [1].
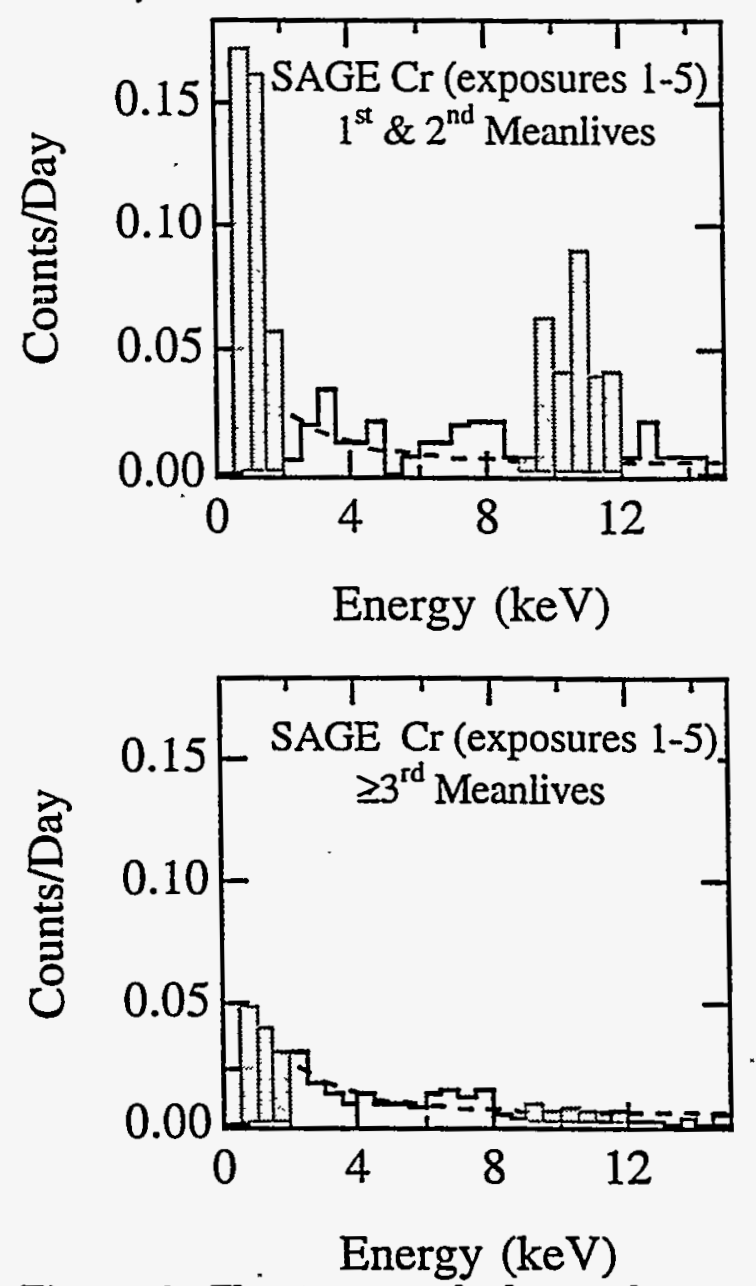

Figure 3. The top panel shows the energy spectrum of the fast risetime events observed during the $5 \mathrm{Cr}$ exposure measurements during the first two meanlives of counting. The bottom panel show a similar spectrum but for counting after two meanlives. The ${ }^{71_{G e}} \mathrm{~K}$ and $\mathrm{L}$ peaks are evident in the top panel.

Table 1. A summary of the contributions to the systematic uncertainty. The total is taken to be the quadrature sum of the contributions. The statistical uncertainty is $\pm 10 \%$.

\begin{tabular}{lc}
\hline \hline Chem. Extr. Efficiency & $\pm 5.5 \%$ \\
Counting Efficiency & $+2.9,-4.2 \%$ \\
Radon & $-7.7 \%$ \\
Solar Neutrino Subtraction & $\pm 0.3 \%$ \\
Carryover Ge Subtraction & $\pm 1.6 \%$ \\
\hline Total Systematic & $+6.4,-10.5 \%$ \\
\hline \hline
\end{tabular}




\section{DISCUSSION}

The final result can be expressed as a ratio of the measured ${ }^{71} \mathrm{Ge}$ production to that expected due to the source strength. The result of 0.93 $+0.15,-0.17$ is consistent with 1.0 to first order. Although a finer level of precision will be determined as the final analysis is completed, the result indicates that the experimental efficiencies are as previously claimed [1]. One concludes therefore that the discrepancy between the SAGE measured solar neutrino flux and the solar model predication cannot be explained by an experimental artifact.

The experimental efficiencies are known to a higher precision than can be tested by this method. Thus in an absolute sense, this measurement does not represent a direct calibration. A more precise description is that the $\mathrm{Cr}$ experiment is a systematic test of the experimental procedures. The solar flux measurement will not be scaled by the above ratio.

GALLEX has completed a ${ }^{51} \mathrm{Cr}$ calibration measurement with the result $0.97 \pm 0.11$ [17]. Both of these experiments similar solar neutrino flux results and have verified their efficiencies even though they employ very different chemistries, indicating that it is very difficult to dismiss the experimental results and their striking disagreement with standard solar model predictions.

\section{ACKNOWLEDGMENTS}

The SAGE collaboration wishes to thank A. E. Chudakov, M. A. Markov, V. A. Matveev, V. A. Rubakov, and A. N. Tavkhelidze for their continued interest in out work and for stimulating discussions. We are also grateful to R. G. H. Robertson, A. Yu. Smirnov, and many members of the GALLEX collaboration for useful discussions. We acknowledge the support of the Russian Academy of Sciences, the Institute for Nuclear Research of the Russian Academy of Sciences, the Russian Ministry of Science and Technology, the Russian Foundation of Fundamental Research, the Division of Nuclear Physics of the Department of Energy, and the National Science
Foundation. The research described in this publication was made possible in part by Grant M7F000 from the International Science Foundation and Grant M7F300 from the International Science Foundation and Russian Goverment.

\section{REFERENCES}

* present address: Department of Particle and Nuclear Physics, Oxford University, Keble Road, Oxford OX1 3RH, UK.

1. J. N. Abdurashitov et al., Phys. Lett. B357, 237 (1995); and these proceedings.

2. P. Anselmann et al., Phys. Lett. B327, 377 (1994).

3. J. N. Bahcall, and M. Pinsonneault, Rev. Mod. Phys., Oct. 1995, in press.

4. S. Turck-Chieze and I. Lopes, Astrophys. J. 408, 347 (1993).

5. B. T. Cleveland et al., Nucl. Phys. (proceed. supl.) B38, 47 (1995).

6. Y. Suzuki, Nucl. Phys. (proceed. supl.) B38, 54 (1995).

7. J. N. Bahcall, Phys. Lett. B338, 276 (1994).

8. V. Berezinsky, Comm. Nucl. Part. Phys. 21, 249 (1994).

9. S. Parke, Phys. Rev. Lett. 74, 839 (1995).

10. N. Hata, S. Bludman, and P. Langacker, Phys. Rev. D49, 3622 (1994).

11. V. Castellani et al., Phys. Rev. D50, 4749 (1994).

12. U. Schotzig and H. Schrader, Halbwertszeiten und PhotonenEmissions-wahrscheinlichkeiten von haufig verwendeten Radionukliden, PTB-Bericht PTB-Ra-16/4, Braunschweig, 1993.

13. J. N. Bahcall and R. K. Ulrich, Rev. Mod. Phys. 60, 297 (1988).

14. Naoya Hata and Wich Haxton, Phys. Lett. B353, 422 (1995).

15. S. R. Elliott, Nucl. Instrum. and Meth. A290, 158 (1990)

16. B. T. Cleveland, Nucl. Instrum. Methods 214, 451 (1983).

17. P. Anselmann et al., Phys. Lett. B342, 440 (1995); and these proceedings. 


\section{DISCLAIMER}

This report was prepared as an account of work sponsored by an agency of the United States Government. Neither the United States Government nor any agency thereof, nor any of their employees, makes any warranty, express or implied, or assumes any legal liability or responsibility for the accuracy, completeness, or usefulness of any information, apparatus, product, or process disclosed, or represents that its use would not infringe privately owned rights. Reference herein to any specific commercial product, process, or service by trade name, trademark, manufacturer, or otherwise does not necessarily constitute or imply its endorsement, recommendation, or favoring by the United States Government or any agency thereof. The views and opinions of authors expressed herein do not necessarily state or reflect those of the United States Government or any agency thereof. 